\title{
Fiber-Optic True Time Steering of an Ultrawide-Band Receive Array
}

\author{
Michael Y. Frankel, Paul J. Matthews, and Ronald D. Esman, Senior Member, IEEE
}

\begin{abstract}
We report the development and demonstration of the first true time-delay (TTD) fiber-optic beamformer for ultrawideband antenna receive operation. The beamformer is based on a dispersive-fiber prism configuration that properly time delays the signals originating at each receiving element for coherent combining in the microwave domain. The time-steered receive antenna consists of eight spiral elements arranged into a onedimensional (1-D) sparsely populated array. The receive array shows an instantaneous bandwidth of 6-18 GHz, limited by the available matched microwave amplifiers. The array performance is measured in an anechoic chamber and exhibits unprecedented squint-free steering over an azimuth scan of $120^{\circ}$ over the full frequency range.
\end{abstract}

Index Terms - Antenna array feeds, phased arrays, optical control, optical fiber delay lines.

\section{INTRODUCTION}

$\mathbf{T}$ HE field of phased-array antenna development is seeing rapid growth. Motivation for the increased efforts is provided by a number of advantages shown by electronically steered array antennas over their mechanically steered counterparts. Among others, these advantages include faster steering speed, improved reliability, and added functionality such as active beamshaping and control. One of the more important functions being given special attention is the implementation of frequency-independent (wide-band) steering, which requires true time-delay (TTD) control of individual array elements. So far, all-electronic methods have proven inadequate for achieving TTD control, and a number of optical control methods have been explored as possible solutions [1]-[6]. While promising, the optical control methods have generally not lived up to their expectations due to the practical limitations imposed by specialized component availability, precision matching and alignment, limited bandwidth, signal loss, and/or excessive power requirements. Recently, we have developed and demonstrated one-dimensional (1-D) [7] and two-dimensional (2-D) [8] ultrawide-band time-steered array (TSA) transmitters controlled by a novel dispersive-fiber-based beamformer. While optically controlled wide-band receive antennas have been demonstrated [9], [10], ultrawide-band receive-mode operation covering more than $10 \mathrm{GHz}$ has never been previously demonstrated.

Manuscript received December 2, 1996; revised April 14, 1997. This work was supported by the Office of Naval Research under the Advanced Capabilities Initiative Program.

The authors are with the Naval Research Laboratory, Optical Sciences Division, Washington, DC 20375 USA.

Publisher Item Identifier S 0018-9480(97)05985-1.

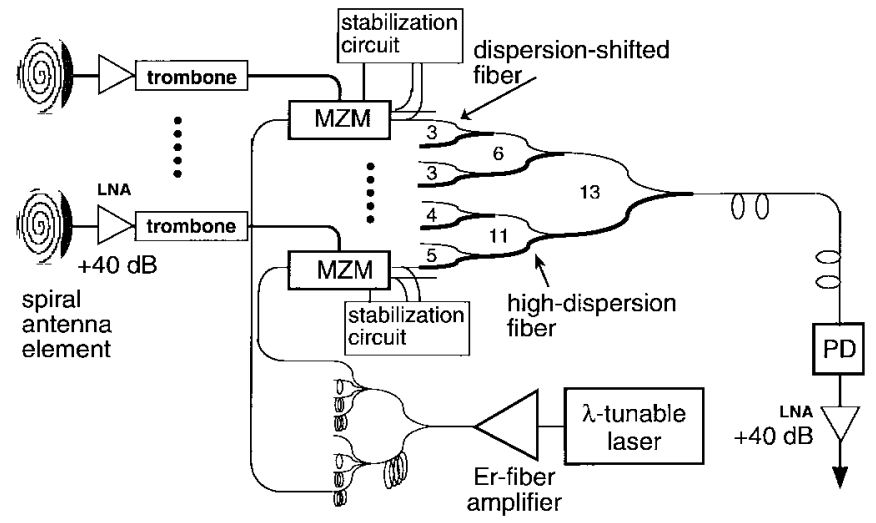

Fig. 1. Fiber-optic TTD beamformer receiver configuration.

Here we extend the above work to develop and demonstrate a receive-mode operation of an ultrawide-band TSA antenna. The antenna consists of eight broad-band spiral elements arranged into a sparsely populated linear array. The array bandwidth was defined by the available 6-18$\mathrm{GHz}$ microwave amplifiers. In time-steered receive mode, the array demonstrates squint-free $\pm 60^{\circ}$ azimuth steering across the full $6-18-\mathrm{GHz}$ frequency range. To our knowledge, this is a first demonstration of an optically controlled receive array in this frequency range, and a first demonstration of an array receive function across such wide instantaneous bandwidth.

\section{FibER-OPTIC BEAMFORMER CONFIGURATION AND CHARACTERISTICS}

\section{A. Beamformer Configuration}

Our antenna consisted of eight broad-band spiral elements arranged into a sparsely populated linear array. The individual elements were absorber-backed spirals with excellent radiation characteristics covering the $2-18-\mathrm{GHz}$ frequency range. The array elements were spaced by 7.5, 7.5, 7.5, 10, 10, 17.5, and $12.5 \mathrm{~cm}$, respectively, with the smallest unit array-spacing increment $d_{\text {unit }}$ being $2.5 \mathrm{~cm}$. In comparison to an eightelement array with conventional half-wavelength spacing, this design reduces the main lobe width, improving angular discrimination, while at the same time suppressing grating lobe formation across a large frequency range. The design tradeoff is an increase in the sidelobe level far away from the main lobe. 
Fig. 1 shows a diagram of the fiber-optic beamformer. The beamformer design is based on a modification of a dispersivefiber architecture previously described for a 1-D transmitter [7]. Following the optical signal path in Fig. 1, the optical source was a commercially available semiconductor laser tunable across the 1535-1565-nm wavelength range. ${ }^{1}$ The linearly polarized optical output was split by a 1:8 polarizationmaintaining splitter network and used to feed eight nominally matched wide-band electro-optic Mach-Zehnder modulators (MZM's). The modulators were driven by the corresponding array antenna elements, with signals amplified by eight broadband microwave low-noise amplifiers (LNA's). The LNA's were nominally matched to $\pm 2 \mathrm{~dB}$ in gain and to $\pm 10^{\circ}$ in phase across the $6-18-\mathrm{GHz}$ frequency range, had a gain of $\sim 40 \mathrm{~dB}$, and served to improve the dynamic range of the fiber-optic links [11]. The output of each MZM was fed into an eight-branch binary-tree fiber-optic dispersive prism. The fiber lengths in each tree branch were designed such that the path traversed by each optical signal possessed optical dispersion proportional to the corresponding-element position within the array by including high-dispersion (HD) fiber with a dispersion of $D_{\mathrm{hd}} \sim-87 \mathrm{ps} / \mathrm{nm} \cdot \mathrm{km}$. Hence, tuning the wavelength of the optical carrier signal modified its group velocity in the link and imposed a time-delay difference linearly related to the product of wavelength detuning and total link dispersion. The unit length of fiber used was $\ell_{\text {unit }}=55 \mathrm{~m}$, and the number of fiber unit lengths included into each branch is indicated in Fig. 1. All fiber-optic links were equalized in length to within $\pm 5 \mathrm{~mm}$ with dispersion shifted $(D \sim 0 \mathrm{ps} / \mathrm{nm} \cdot \mathrm{km})$ fiber.

After all eight signals are incoherently combined in the optical domain (discussed in more detail below), the combined signal is demodulated by a $50-\Omega$ matched $p-i-n$ photodetector (PD) and amplified by a broad-band microwave amplifier.

While the MZM's were nominally fabricated for a nearzero dc-bias voltage at quadrature, we used active dc-bias stabilization to insure the long-term stability of the system. Approximately $1 \%$ of the optical-power output from each complementary MZM optical output was tapped off and fed to a stabilization circuit. The circuit was designed to preserve optimal dc bias by maintaining equal optical power in each of the MZM outputs.

Various implementations of the fiber-optic dispersive prism are possible. The most basic uses individual fiber-optic links with a separate PD for each array element. This approach has the advantages of efficient optical-power utilization and of naturally avoiding coherent optical interference effects because the signal summation is carried out in the microwave domain. However, the drawbacks include the requirements for a PD for each array element and large amount of expensive high dispersion fiber. We have chosen a design based on a binarytree architecture that uses incoherent optical combining. Thus, we only require a single PD for the complete beamformer. The amount of HD fiber is also reduced by a factor of $\sim 2.2$. The optical interference effects are reduced by introducing a

\footnotetext{
${ }^{1}$ Photonetics, Inc., Model Tunics 1550 tunable external cavity laser, Photonetics, Wakefield, MA.
}

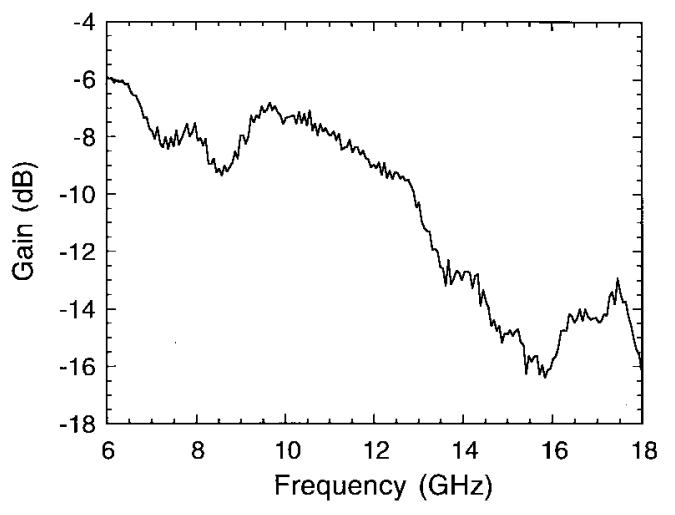

Fig. 2. Single beamformer-link frequency response.

length differential between the branches of the 1:8 opticalpower splitter that is greater than the coherence length of the laser. The length difference of $\sim 1.3 \mathrm{~m}$ was verified to be sufficient to minimize the interference effects when used with the laser coherence control which broadened the linewidth to $>100 \mathrm{MHz}$.

The complete fiber-optic beamformer (excluding the laser source and the array elements) was packaged into a standard $8^{\prime \prime}$-high $19^{\prime \prime}$ instrument rack box.

The relationship between the laser detuning wavelength $\Delta \lambda$ and the corresponding array steering-angle $\Theta$ is given as

$$
\Theta=\sin ^{-1}\left(\frac{c \cdot \ell_{\text {unit }} \cdot D_{\text {hd }}}{d_{\text {unit }}} \Delta \lambda\right)
$$

where $c$ is the speed of light, $\ell_{\text {unit }}$ is the smallest unit increment of the HD fiber, $D_{\text {hd }}$ is the dispersion of the HD fiber, and $d_{\text {unit }}$ is the smallest unit increment element spacing.

For our system, the following were used: $\ell_{\text {unit }}=55 \mathrm{~m}$, $D_{\text {hd }}=-87 \mathrm{ps} / \mathrm{nm} \cdot \mathrm{km}$, and $d_{\text {unit }}=0.025 \mathrm{~m}$ giving (with $\Delta \lambda$ in $\mathrm{nm}$ )

$$
\Theta=\sin ^{-1}(0.057 \Delta \lambda)
$$

\section{B. Beamformer Benchtop Characteristics}

Prior to anechoic chamber tests, the beamformer was characterized on the benchtop without the array elements. In particular, aspects such the frequency response, dynamic range, and interchannel amplitude and phase tracking were investigated.

A network analyzer was used to measure the beamformer frequency-domain characteristics. Fig. 2 shows a typical frequency response with only one of the channels being fed with the network analyzer. The beamformer shows a single-channel insertion loss of $\sim 4 \mathrm{~dB}$ at the lower frequency range. This is consistent with the $G_{\mathrm{LNA}} \sim 40$-dB front-end LNA gain, MZM $V_{\pi}$ of $\sim 15 \mathrm{~V}$, an average PD photocurrent $I_{\mathrm{PD}}$ (from all eight channels) of $\sim 100 \mu \mathrm{A}, 50-\Omega \mathrm{PD}$ matching, and a $G_{\mathrm{OA}} \sim 40$-dB gain from the output amplifier based on

$G_{\mathrm{ch}}=-22+20 \log \left(V_{\pi}\right)-20 \log \left(I_{\mathrm{PD}}\right)+G_{\mathrm{LNA}}+G_{\mathrm{OA}}$. 


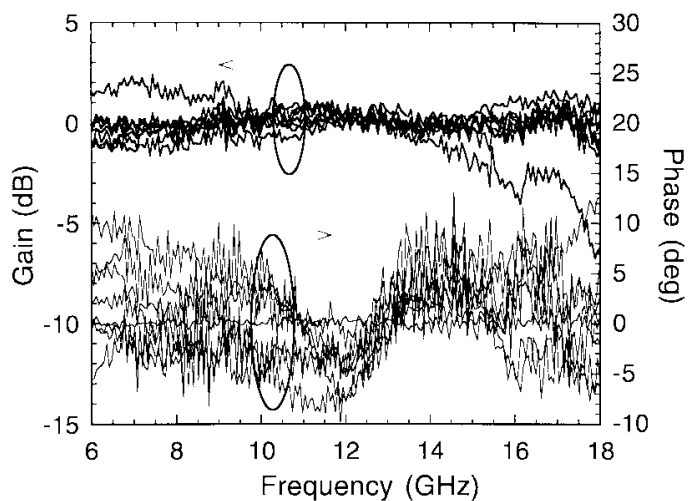

Fig. 3. Amplitude and phase tracking among the eight individual links.

It should be noted that switching to individual fiber-optic links and microwave combining would reduce the insertion loss by $\sim 18 \mathrm{~dB}$ through elimination of the optical losses associated with incoherent combining [i.e., factor of $20 \log (8)$ ]. This architecture may be preferable for a production system if the added cost of the additional PD's is acceptable.

The signal-to-noise ratio (SNR) for our system was measured to be $87 \mathrm{~dB} / \mathrm{Hz}$. Using an optimized fiber-optic link with a commercially available MZM modulator with a low $V_{\pi}$ of 5 $\mathrm{V},{ }^{2}$ the SNR can be improved to $\sim 150 \mathrm{~dB} / \mathrm{Hz}$ with a spuriousfree dynamic range of over $100 \mathrm{~dB}$ in a $1-\mathrm{Hz}$ bandwidth, as calculated based on the analysis in [11].

An important parameter for a wide-band array system is the amplitude and phase tracking of the individual link responses across the full instantaneous bandwidth. Fig. 3 shows the amplitude and phase tracking between the individual links. The first link was taken as a reference by calibrating the network analyzer throughput on its response. We observe good amplitude tracking with deviations bounded by $\pm 2 \mathrm{~dB}$ across the full $6-18-\mathrm{GHz}$ frequency range with the exception of one channel. The errant response of this channel is believed to be due to the poor response of the microwave amplifier in that channel. The phase tracking exhibited deviations bounded by $\pm 10^{\circ}$ across the full $6-18-\mathrm{GHz}$ frequency range. The overall system amplitude and phase deviations were traced primarily to the microwave amplifiers.

\section{ReCEIVE ARRAY OPERATING Characteristics}

The array antenna receive patterns were measured in an anechoic chamber. A network analyzer was used to drive a radiating broad-band horn element. The radiation was collimated by a microwave mirror and detected by the receive array. The single output of the fiber-optic beamformer was fed back to the network analyzer for processing. The receive array, along with the fiber-optic beamformer, was positioned on a rotation tower. Azimuth scans were taken across the $\pm 70^{\circ}$ range in $0.25^{\circ}$ increments, with frequencies ranging from 6 to $18 \mathrm{GHz}$ in $1-\mathrm{GHz}$ increments.

Initially, we measured single-element patterns at the stated frequencies. These patterns were subsequently used to normalize the measured array patterns, thereby providing a better picture of the intrinsic array properties. An array-factor pattern,

\footnotetext{
${ }^{2}$ Sumicem Optoelectronics, Ltd., Limerick, Ireland.
}

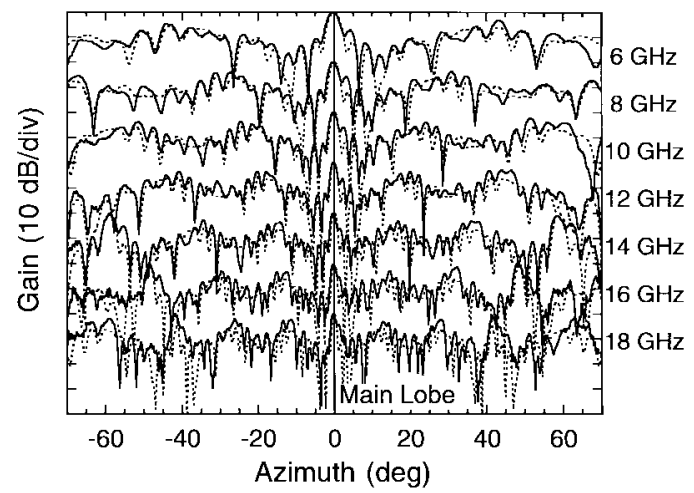

Fig. 4. Comparison between the normalized measured (solid) and ideal calculated (dashed) antenna receive patterns with the laser tuned for broadside radiation $(\Delta \lambda=0 \mathrm{~nm})$. Frequencies of $6,8,10,12,14,16$, and $18 \mathrm{GHz}$ are shown with offsets for clarity.

calculated assuming ideal array receive elements with no mutual coupling, provides a convenient benchmark for evaluating our receiver performance.

Fig. 4 shows the measured and the calculated (ideal) receive-array antenna patterns with the laser set at $1550 \mathrm{~nm}$ for optical broadside steering. As expected, we observe a main lobe steered to broadside and the lobe width narrowing as the frequency increases from 6 to $18 \mathrm{GHz}$. In accordance with the array design, close $\left( \pm 10^{\circ}\right)$ to the main lobe the sidelobe level is $\sim 15 \mathrm{~dB}$ below the main lobe, whereas far $\left(>20^{\circ}\right)$ from the main lobe the sidelobes increase to a level of only $\sim 5 \mathrm{~dB}$ below the main lobe. The advantage of this design is a narrow main-lobe width and an absence of grating lobes at all frequencies up to $13 \mathrm{GHz}$.

The agreement between the measured and calculated patterns is good over the entire frequency and azimuth range. This indicates that the amplitude and phase match among the eight receive channels measured in Fig. 3 does not appreciably degrade our array patterns. Furthermore, the element mutual coupling appears to be negligible in our array possibly due to the large inter-element separation.

The true power of the TSA architecture appears when the array is steered off broadside. The fiber-prism-based array is steered simply by changing the wavelength of the optical carrier.

The angular steering limit in our system is determined primarily by the optical bandwidth of the Er-fiber amplifier. The Er-amplifier bandwidth allowed a wavelength detuning of at most $\pm 15 \mathrm{~nm}$, before significant optical-power reduction occurred. From (2), this corresponds to an angular steering range of $\pm 60^{\circ}$.

Fig. 5 shows the array patterns measured with the laser wavelength tuned to $1535 \mathrm{~nm}(\Delta \lambda=-15 \mathrm{~nm})$, which corresponds to a steering angle of $-60^{\circ}$. Squint-free steering over our operating bandwidth is observed with a good match between the measured and calculated array patterns, which indicates negligible wavelength-dependent effects in the system Similarly, we verified that the array could be steered to other angles within the $\pm 60^{\circ}$ azimuth range with no measurable squint and with good agreement between the measured and calculated array patterns. 


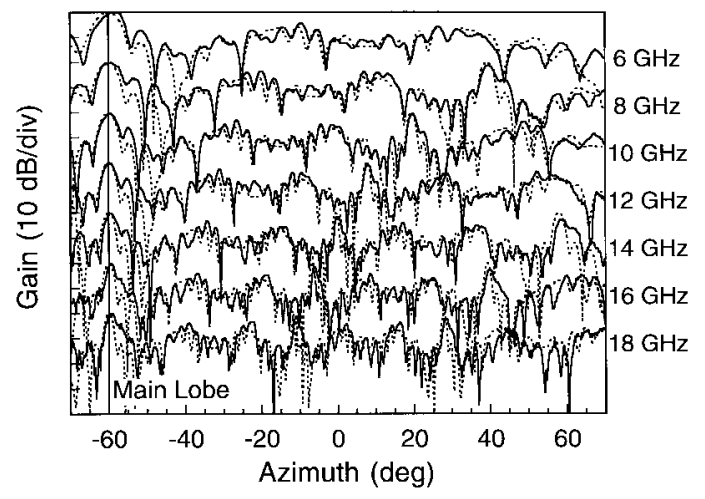

Fig. 5. Comparison between the normalized measured (solid) and ideal calculated (dashed) antenna receive patterns with the laser tuned for $-60^{\circ}$ radiation $(\Delta \lambda=-15 \mathrm{~nm})$. Frequencies of $6,8,10,12,14,16$, and $18 \mathrm{GHz}$ are shown with offsets for clarity.

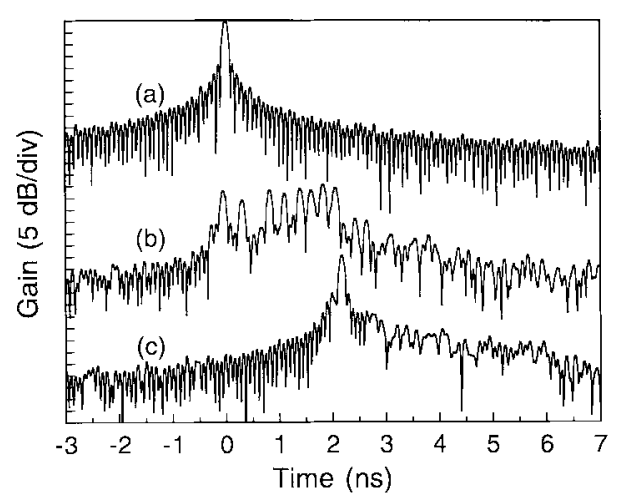

Fig. 6. Synthesized time-domain receiver impulse response with antenna mechanical- and optical-steering settings as parameter. $a: \Delta \lambda=0 \mathrm{~nm}$, mechanical broadside, $b: \Delta \lambda=0 \mathrm{~nm}$, mechanical steering to $-60^{\circ}, c$ : $\Delta \lambda=-15 \mathrm{~nm}$, mechanical steering to $-60^{\circ}$.

\section{TIME-DOMAIN IMPUlse ResPONSE}

More intuitive insight into the system operation can be gained in the time domain, where the wide instantaneous bandwidth of the receiver can be used to demonstrate a fast pulse response. The available measurement equipment allowed us to measure only the frequency response as a function of the various mechanical and optical steering angles. However, since our system is operating in the linear regime [7], we can apply an inverse Fourier transform to obtain the equivalent time-domain response.

Fig. 6 shows the time-domain responses obtained for several antenna mechanical- and optical-steering settings. The response of the microwave components has been calibrated out using a single channel throughput as a normalization reference. The top curve $a$ corresponds to the array steered to broadside both mechanically and optically. The individual microwave pulses detected by each antenna element are coherently combined to produce a single well-defined output pulse. Then, the array is mechanically rotated by $-60^{\circ}$ while the optical steering is still set for broadside, with the results shown by curve $b$. The signal arrival times at each receiver element are now a function of the element position within the array. Eight pulses are observed at the receiver output with the relative time delays proportional to the corresponding element separation in the array. When the array is optically steered to match the mechanical steering angle by setting the wavelength to $1535 \mathrm{~nm}(\Delta \lambda=-15 \mathrm{~nm})$, the optical paths are effectively adjusted to compensate for the time-of-arrival differences between the individual signals. Curve $c$ now shows that all eight microwave pulses are again coherently added into a single pulse, with its position shifted due to the onesided delay operation of the dispersive fiber links. These results demonstrate the ultrawide-band receive capability with $\sim 100$ ps time-domain resolution, which corresponds to a $3-\mathrm{cm}$ spatial resolution. This resolution is still determined by the microwave amplifiers and is not the fundamental limit of the fiber-optic beamformer.

\section{CONCLusion}

We have developed and demonstrated an ultrawide-band beamformer for array-receive operation. The system is based on an original fiber-optic dispersive prism architecture, which uses only commercially available components. The beamformer possesses all the beneficial characteristics of a fiberoptic system including light weight and compact size while at the same time avoiding the constraints imposed by specialized component development. The beamformer is used to steer an eight-element sparsely populated linear-array antenna in the receive mode. The measurements demonstrate the ultrawideband capability covering the $6-18 \mathrm{GHz}$ frequency range, which is limited by the available matched microwave amplifiers. Completely squint-free steering is demonstrated over the $\pm 60^{\circ}$ azimuth range. The wide instantaneous receiver bandwidth was illustrated by synthesizing the time-domain response of the system which demonstrates 100-ps time resolution.

\section{ACKNOWLEDGMENT}

The authors would like to thank M. G. Parent and J. Valenzi for help with the antenna-pattern measurements.

\section{REFERENCES}

[1] H. Zmuda and E. N. Toughlian, Photonic Aspects of Modern Radar. Norwood, MA: Artech House, 1994.

[2] D. Dolfi, F. Michel-Gabriel, S. Bahn, and J. P. Huignard, "Twodimensional optical architecture for time-delay beam forming in a phased-array antenna," Opt. Lett., vol. 16, no. 4, pp. 255-257, Feb. 1991.

[3] N. A. Riza, "Transmit/receive time-delay beam-forming optical architecture for phased-array antennas," Appl. Opt., vol. 30, no. 32, pp. 4594-4595, Nov. 1991.

[4] A. P. Goutzoulis, D. K. Davies, and J. M. Zomp, "Hybrid electronic fiber optic wavelength-multiplexed system for true time-delay steering of phased array antennas," Opt. Eng., vol. 31, no. 11, pp. 2312-2322, Nov. 1992.

[5] R. Soref, "Optical dispersion technique for time-delay beam steering," Appl. Opt., vol. 31, no. 35, pp. 7395-7397, Dec. 1992.

[6] I. Frigyes and A. J. Seeds, "Optically generated true-time delay in phased array antennas," IEEE Trans. Microwave Theory Tech., vol. 43, pp. 2378-2386, Sept. 1995.

[7] M. Y. Frankel and R. D. Esman, "True time-delay fiber-optic control of an ultrawide-band array transmitter/receiver with multibeam capability," IEEE Trans. Microwave Theory Tech., vol. 43, pp. 2387-2394, Sept. 1995. 
[8] M. Y. Frankel, P. J. Matthews, and R. D. Esman, "Two-dimensional fiber-optic control of a true time-steered array transmitter," IEEE Trans. Microwave Theory Tech., vol. 44, pp. 2696-2702, Dec. 1996.

[9] A. P. Goutzoulis, J. M. Zomp, B. K. Davies, I. Liberman, A. H. Johnson, and P. D. Hricak, "Hardware compressive, true time-steering for control of phased array antennas," Rome Lab. Rep. RL-TR-95-293, Jan. 1996.

[10] J. J. Lee, R. Y. Loo, S. Livingston, V. I. Jones, J. B. Lewis, H. W Yen, G. L. Tangonan, and M. Wechsberg, "Photonic wide-band array antennas," IEEE Trans. Antennas Propagat., vol. 43, pp. 966-982, Sept. 1995.

[11] A. M. Yurek, S. W. Merritt, and G. Drake, "Determining the cascade parameters of externally modulated links," Microwave J., pp. 80-86, Aug. 1995.

Michael Y. Frankel, for a biography, see this issue, p. 1374.

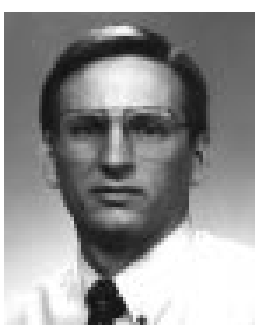

Paul J. Matthews received the B.S. degree (magna cum laude) in physics from Loyola University of Chicago, Illinois, in 1986, and the M.S. and Ph.D. degrees in electrical engineering from the University of Colorado at Boulder, in 1988 and 1991, respectively.

From January 1992 to October 1992, he was employed by CAI/Recon Optical. In October 1992 he joined the staff of the Advanced Photon Source, Argonne National Laboratory, where he was engaged in efforts to design, model, and fabricate a mm-wave linear accelerator using deep-etch x-ray lithography techniques and electroplating. Since September 1995, he has been with the Optical Sciences Division of the Naval Research Laboratory, Washington, DC. His current interests include optical control schemes for phased-array antenna applications and high-performance fiber-optic links.

Ronald D. Esman (S'82-M'85-SM'95), for a photograph and biography, see this issue, p. 1278. 